\title{
Ear Recognition using Dual Tree Complex Wavelet Transform
}

\author{
Rajesh M Bodade \\ Military College of Telecommunication Engineering \\ Mhow, Indore, India
}

\author{
Sanjay N Talbar \\ S.G.G.S. Institute of Technology and Engineering \\ Nanded, India
}

\begin{abstract}
Since last 10 years, various methods have been used for ear recognition. This paper describes the automatic localization of an ear and it's segmentation from the side poses of face images. In this paper, authors have proposed a novel approach of feature extraction of iris image using 2D Dual Tree Complex Wavelet Transform (2D-DT-CWT) which provides six sub-bands in 06 different orientations, as against three orientations in DWT. DT-CWT being complex, exhibits the property of shift invariance. Ear feature vectors are obtained by computing mean, standard deviation, energy and entropy of these six sub-bands of DT-CWT and three sub-bands of DWT. Canberra distance and Euclidian distance are used for matching. This method is implemented and tested on two image databases, UND database of 219 subjects from the University of Notre Dame and own database created at MCTE, of 40 subjects which is also used for online ear testing of system for access control at MCTE. False Acceptance Rate (FAR), False Rejection Rate (FRR), Equal Error Rate (EER) and Receiver's Operating Curve (ROC) are compiled at various thresholds. The accuracy of recognition is achieved above $97 \%$.
\end{abstract}

Keywords-Ear recognition; ear detection; ear biometrics; DTCWT; complex wavelet transform; Biometrics; Pattern Recognition; Security; Image Processing; Bioinformatics; Computer vision.

\section{INTRODUCTION}

Ear recognition has received considerably less attention than many alternative biometrics, including face, fingerprint and iris recognition. Ear-based recognition is of particular interest because it is non-invasive, and also is not affected by other factors such as mood, health, and/or clothing. Also, the appearance of an auricle (outer ear) is relatively unaffected by ageing, making it better suited for long-term identification[1]. Also, ear images can be easily taken from a distance without knowledge of the person concerned. Therefore ear biometric is suitable of surveillance, security, access control and monitoring applications.

As compared to face biometrics [2]-[4] ears have several advantages over complete face like reduced spatial resolution, a more uniform distribution of color, and less variability with expressions and orientation of the face. It's deep threedimensional structure makes it very difficult to counterfeit. Moreover, in face recognition there can be problems of illumination variation, pose variation and facial expressions [4].

Ear was first used for recognition of human being by Iannarelli, who used manual techniques to identify ear images.
Samples of over 10,000 ears were studied to prove the distinctiveness of ear. Structure of ear does not change radically over time. The medical literature provides information that ear growth is proportional after first four months of birth and changes are not noticeable in the age group from 8 to 70 [1].

Thus, this ensures that the ear will occupy a special place in conditions requiring a high degree of perfection.

The remainder of this paper consists of: existing ear recognition techniques, localiasition and normalistion of ear, feature extraction using DT-CWT, matching, experimental results and conclusions covered in Section-2, 3, 4, 5, 6 and 7 respectively.

\section{EXISTING EAR RECOGNITION TECHNIQUES}

Major work on automatic ear localistion [2]-[11] has been done recently in past 10 years. Automatic ear recognition using Voronoi diagrams to take care of adverse effects of lighting, shadowing and occlusion has been presented by Burge and Burger [6]. In [9], Active Contour Model (or Snakes) is used to segment the ear from the side image of a face. Hurley, Nixon and Carter [7] have used force field transformations for ear localisation. [8][12] and [13] make use of 3-D range images to extract the ear from the image of a human. However, the tougher challenge is to detect the ear from an intensity image. A shaped model-based technique for locating human ears from face range images is proposed in [8]. In this method, the ear shape model is represented by a set of discrete 3D vertices corresponding to ear's helix and anti-helix parts. Ansari and Gupta have proposed an approach based on edges of outer ear helices by exploiting the parallelism between the outer helix curves of the ear to localize the ear[10]. Skin-color and contour information has been exploited for ear detection by Yuan and $\mathrm{Mu}$ [11]. In [13], authors has presented a distance transform and template based technique for automatic ear localization from a side face image. This technique first segments skin and non-skin regions in a face and then uses template based approach to find the ear location within the skin regions.

Victor et al. [4] and Chang et al. [2] have researched use of PCA and FETET for ear recognition. Moreno et al. [5] used 2D intensity images of ears with three neural net approaches for ear recognition. Hurley [14] developed an invertible linear transform which transforms an ear image into a force field by pretending that pixels have a mutual attraction proportional to 
their intensities and inversely proportional to the square of the distance between them. Yan and Bowyer [15] have developed a fully automatic ear biometric system using ICP based 3D shape matching for recognition, and using both 2D appearance and 3D depth data for automatic ear extraction which not only extracts the ear image but also separates it from hair and earrings. In [16], Anupama Sana et al. presented an ear biometric system based on discrete Haar Wavelet Transform whereas Wang and Yuan [17] used Gabor wavelets and general discernment analysis. Wang Xiaoyun et al. [19] proposed block segmentation based approach whereas modular neural network architecture has been proposed by Gutierrez et al. [18].

\section{PROPOSED SYSTEM}

The block diagram of proposed system is shown in Fig 1. It consist of an image acquisition module, preprocessing and automatic ear localization module, DT-CWT based feature extraction module and matching module.

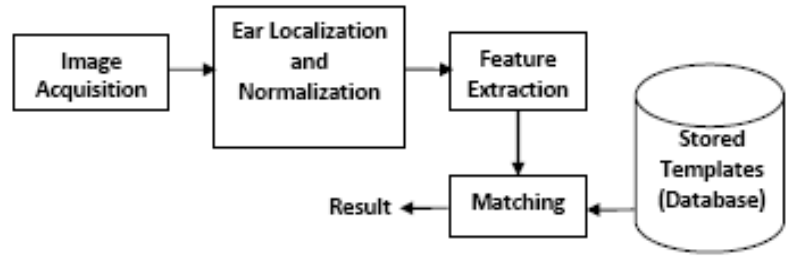

Figure 1. Block diagram of proposed system

\section{A. Image Acquisition Module}

Sony DSC-HX1 (15 Megapixel and optical zoom of 20x). camera is used for image acquisition. Database of 240 images of 40 subjects for left and right ears created at MCTE and UND database is also used.

\section{B. Preprocessing and ear localisation module}

The raw image is not suitable for feature extraction due to its large background. Thus some pre-processing is required to make it suitable. The important steps involved are: gray scale conversion, ear detection and scale normalization which has been presented in section III .

\section{Feature extraction module}

After successful ear localization, features are extracted using DT-CWT. The details of the same are stated in section IV.

\section{Matching module}

Energy, Entropy, Mean and Standard Deviation of each subband of DT-CWT is calculated to create a feature vector. Euclidian distance and Canbera distance as given by equation (1) and (2) are used as similarity measures for matching the feature vectors of the test image with that of the images stored in database $(1: \mathrm{N})$ match.

Euclidian

$$
\mathrm{d}_{\mathrm{E}}\left(\mathrm{x}_{\mathrm{y}} \mathrm{y}\right)=\sqrt{\sum_{\mathrm{i}=1}^{\mathrm{d}}\left(\mathrm{x}_{\mathrm{i}}-\mathrm{y}_{\mathrm{i}}\right)^{2}}
$$

Distance:

\section{Canbera Distance:}

$$
\mathrm{d}_{\mathrm{c}}\left(\mathrm{x}_{y} \mathrm{y}\right)=\sum_{\mathrm{i}=1}^{\mathrm{d}} \frac{\| \mathrm{x}_{\mathrm{i}}-\mathrm{y}_{\mathrm{i}} \mid}{\left|\mathrm{x}_{\mathrm{i}}\right|+\left|\mathrm{y}_{\mathrm{i}}\right|}
$$

\section{AutOMATIC EAR LOCALISATION}

This was a very challenging task as most of the work carried out on this aspect is in experimental stage. The algorithm so designed includes the finer points of various algorithms and additional measures to try and further enhance and improve the ear localization results. The algorithm works as under.

(i) Take a side face image of an individual (under varied background and lighting conditions).

(ii) Creates the color transformation structure from RGB to LAB and apply it to the test image. The input is RGB which consists of three layers, Red, Green, and Blue. The output is LAB which consists of $\mathrm{L}^{*}, \mathrm{a}^{*}$, and $\mathrm{b}^{*}$ layers. $\mathrm{L}^{*}$ represents brightness and has range from 0 to 100 . $\mathrm{a}^{*}$ represents degree of redness-greenish, having range from -100 to 100. (Positive values for redness, and negative values for greenish). $b^{*}$ represents degree of yellowish-bluish and has the same ranges as $\mathrm{a}^{*}$.

(iii)The technique is adaptable for different skin colors and various lighting conditions. Since $R G B$ representation of color images is not suitable for characterizing skin-color, it first converts the $R G B$ color space to chromatic color space, and then uses the chromatic color information for further processing. In $R G B$ color space, the triple components $(R, G \&$ $B$ ) represent not only color but also luminance which may vary across a person's face due to the ambient lighting and is not a reliable measure in separating skin from non-skin regions. Luminance can be removed from the color representation in the chromatic color space.

(iv)Apply threshold to the resultant image and convert to binary.

(v) A few results under varied background conditions are shown from Figure 2 to Figure 4.

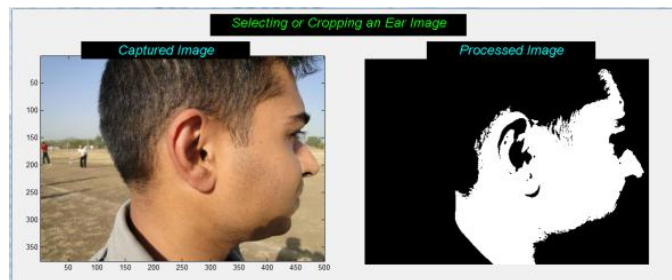

Figure 2. Side face image with open sky background

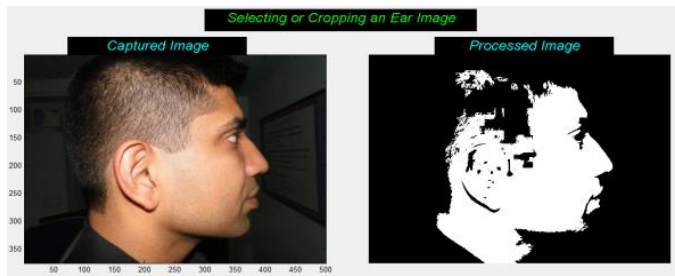

Figure 3. Side face image with class room background 


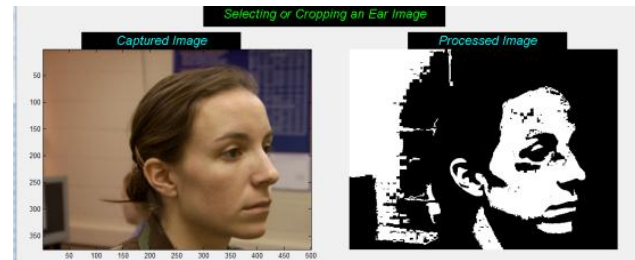

Figure 4. Side face image with class room background from UND database

(vi) Once the background is removed from an image, we determine the nose tip of the subject and assuming an approximate distance between the subject's nose tip \& ear pit and the average size of human ear, we crop the ear part from the side face of an image.

(vii) Thereafter we take a four pronged approach to determine the edge variations from top (at Helix), from side (at Concha and at Helix posterior) and from bottom (at Lobulo) to determine the ear edges and thus crop out the exact ear from an image.

(viii) A two resulting ear image localized automatically from side face images are shown in Figure 5.

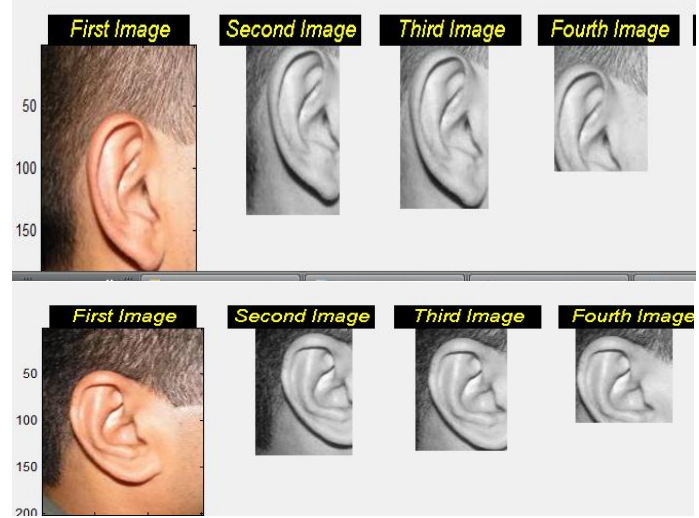

Figure 5. Automatic cropping for Ear localization

(ix) The cropped ear images may be of varying sizes and so the feature set of images may also vary. Hence the images are normalized to a constant size by resizing technique (Bilinear method). If two images are of different sizes, e.g. one is of size $\left(x^{\prime}, y^{\prime}\right)$ and the other is of size (x", $\left.y^{\prime \prime}\right)$, then the two images are mapped to a constant size.

$$
\begin{aligned}
& \mathrm{I}\left(\mathrm{x}^{\prime}, \mathrm{y}^{\prime}\right)=\mathrm{I}(\mathrm{x}, \mathrm{y}) \\
& \mathrm{I}\left(\mathrm{x}^{\prime}, \mathrm{y}^{\prime \prime}\right)=\mathrm{I}(\mathrm{x}, \mathrm{y})
\end{aligned}
$$

\section{FEATURE EXTRACTION}

DT-CWT is formulated by Kingbury and Selesnick[20], [21] using two trees (real and imaginary trees) of DWT with different filter real coefficients for imaginary tree filters designed from the coefficients of real tree filters to overcome the limitations (Poor directionality, shift variance and absence of phase information) of DWTs. Kokare et al. [22] has rotated the 2-D non-separable filters to obtain 2-D non separable Rotated Complex Wavelet Filters (RCWF) and used DT-CWT and RCWF for content based texture image retrieval with excellent retrieval results compared to many other methods. Same concept with different set of filters has been used by
Bodade et al. [23], [24] for iris recognition and obtained a comparable result with the benchmarked Daughman's method[25]. The details of DT-CWT and feature extraction are stated in following subsections.

\section{A. Dual Tree Complex Wavelet Transform}

In dual-tree, two real wavelet trees are used as shown in Figure 6, each capable of perfect reconstruction (PR). One tree generates the real part of the transform while the other is used in generating complex part[20]. As shown, $\{\mathrm{H} 0(\mathrm{z}), \mathrm{H} 1(\mathrm{z})\}$ is a Quadrature Mirror Filter (QMF) pair in the real-coefficient analysis branch. For the complex part, $\{\mathrm{G} 0(\mathrm{z}), \mathrm{G} 1(\mathrm{z})\}$ is an another QMF pair in the analysis branch.

All filter pairs are orthogonal and real-valued. It has been shown [21] that if filters in both trees be made to be offset by half-sample, two wavelets satisfy Hilbert transform pair condition and an approximately analytic wavelet is given by $\mathrm{Eq}(1)$.

$\psi(x)=\psi_{h}(x)+j \psi_{g}(x)$

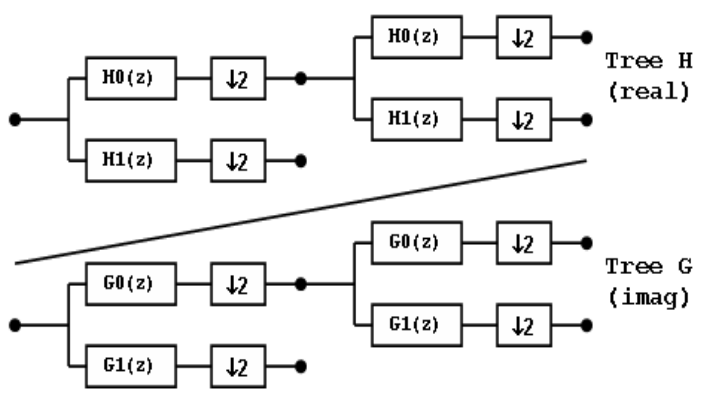

Figure 6. Selesnick's Dual Tree DWT

Thus, if $G_{0}(\omega) \cong H_{0}(\omega) \times e^{-i \theta(\omega)}$ and $\quad \theta(\omega) \cong \omega / 2$

then $\psi_{g}(\omega) \cong-j \psi_{h}(\omega), \omega>0$

$$
\cong j \psi_{h}(\omega), \omega<0
$$

From $\operatorname{Eq}(1)$ and (2), low pass filters after the first stage and at first stage respectively are given by $\mathrm{Eq}(3)$ :

$\mathrm{g}_{0}(\mathrm{n})=\mathrm{h}_{0}(\mathrm{n}-0.5)$ and

$g_{0}(n)=h_{0}(n-1)$

Similar relations also hold true for high pass filters of both the trees.

In this algorithm, $(10,10)$-Tap near orthogonal wavelet filters are used in first stage and ' $\mathrm{db} 7$ ' filters are used for higher stages in the real tree (i.e. $\mathrm{h}_{0}$ and $\mathrm{h}_{1}$ )[20].The imaginary low pass filter is derived from the above half sample delayed condition. The high pass filter is the quadrature-mirror filter of the low pass filter. The reconstruction filters are obtained by time reversal of decomposition filters. All the filters used are of same length based on Selesnick's approach [20], [21], [23], [24] unlike Kingsbury's approach.

The 2D separable DWT can be written in terms of 1D scaling functions $(\varphi)$ and wavelet functions $(\psi)$ as: 


$$
\begin{aligned}
& \psi^{0}(x, y)=\phi(x) \psi(y) \\
& \psi^{90}(x, y)=\psi(x) \phi(y) \\
& \psi^{ \pm 45}(x, y)=\psi(x) \psi(y)
\end{aligned}
$$

Oriented non-separable 2D wavelet transform is derived by combining the sub-bands of two separable 2D DWTs. The pair of conjugate filters are applied to two dimensions ( $\mathrm{x}$ and $\mathrm{y}$ ), which can be expressed by $\operatorname{Eq}(5)$ as given below:

$$
\left(h_{x}+j g_{x}\right)\left(h_{y}+j g_{y}\right)=\left(h_{x} h_{y}-g_{x} g_{y}\right)+j\left(h_{x} g_{y}+h_{y} g_{x}\right)
$$

The filter bank structure of 2D DT CWT, to implement $\mathrm{Eq}(5)$ is shown in Figure 7.

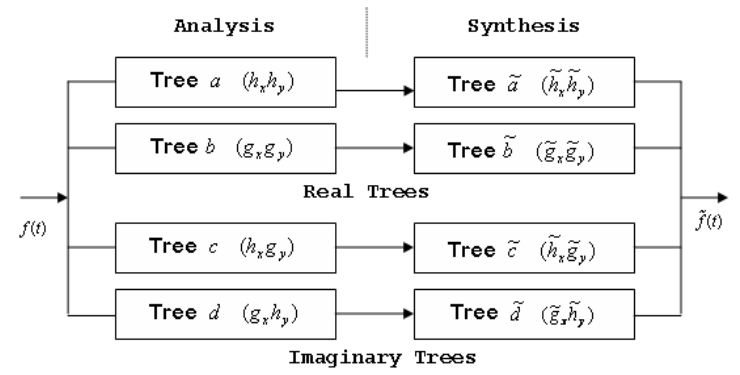

Figure 7. Filter bank structure of 2D DT CWT

Tree-a and Tree-b is combined to compute the Real part of $\mathrm{Eq}(5)$ i.e Real (2D DWT) tree of CWT as shown in Figure 8. Similarly, Imaginary (2D DWT) tree of CWT can be obtained from tree-c and tree-d i.e. $\left(h_{x} g_{y}-g_{x} h_{y}\right)$, as per $\operatorname{Eq}(5)$.

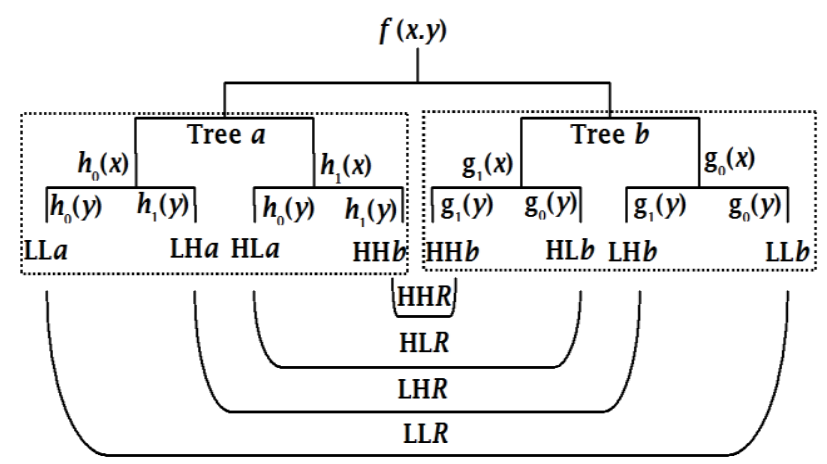

Figure 8: Formation of Real Tree DT CWT

Thus, the decomposition for each mode is performed in a standalone mode, in subsequent stages i.e. total of 6 detailed coefficients are derived at each stage; three each for real and imaginary trees. When 3-stage decomposition is performed, at each stage, coefficients are oriented towards their respective directions as stated in $\mathrm{Eq}(4)$. Following six wavelets, as given by $\mathrm{Eq}(6)$, are used to obtain oriented 2-D separable wavelets [20]:

$\begin{array}{ll}\psi_{1,1}(x, y)=\phi_{h}(x) \psi_{h}(y), & \psi_{2,1}(x, y)=\phi_{g}(x) \psi_{g}(y), \\ \psi_{1,2}(x, y)=\psi_{h}(x) \phi_{h}(y), & \psi_{2,2}(x, y)=\psi_{g}(x) \phi_{g}(y), \\ \psi_{1,3}(x, y)=\psi_{h}(x) \psi_{h}(y), & \psi_{2,3}(x, y)=\psi_{g}(x) \phi_{g}(y),\end{array}$

where, $\psi_{1, \mathrm{i}}$ corresponds to the coefficients derived from the real tree and $\psi_{2, \mathrm{i}}$ corresponds to the coefficients derived from the imaginary tree. They can thus be combined by $\operatorname{Eq}(7)$ to form complex wavelet coefficients.

$$
\begin{aligned}
& \psi_{i}(x, y)=\frac{1}{\sqrt{2}}\left(\psi_{1}, i(x, y)-\psi_{2}, i(x, y)\right), \\
& \psi_{i+3}(x, y)=\frac{1}{\sqrt{2}}\left(\psi_{1}, i(x, y)+\psi_{2}, i(x, y)\right.
\end{aligned}
$$

Normalization by $1 / \sqrt{2}$ is used so that the sum/ difference operation constitutes an ortho-normality. These six wavelet sub-bands of the 2-D DT-CWT are strongly oriented in $\left\{+15^{\circ},+45^{\circ},+75^{\circ},-15^{\circ},-45^{\circ},-75^{\circ}\right\}$ direction as shown in fig $(5)$ by red lines and it captures image information in those directions.

Thus, in particular, 2D dual-tree wavelets are not only approximately analytic but are also oriented and shift invariant because of their analytic structure[20].

The impulse responses of three 2-D sub-bands (2-D non separable filters for detailed coefficients) of DWT and six sub-bands (2-D non separable filters for detailed coefficients) of DT-CWT are shown in Figure 9.

\section{B. Feature Extraction}

Ear analysis using DWT provides singularities (edges) in only three directions $(0,45,90)$ and without phase information which is improved by finding the singularities, with phase information, in six directions $(0,+/-15,+/-30,+/-45,+/-60,+/-$ $75,90)$ and at many freq bands using DT-CWT to achieve shift invariant features for better accuracy and efficiency at less computational cost as compared to existing methods.

From the detailed study of prevalent techniques already employed for ear recognition, it is realized that nobody had made use of Complex Wavelets for ear recognition. This realization laid the foundation of utilizing this approach to determine whether or not the said approach can further enhance and improve the recognition rates already achieved by other methods. As an advantage of using CWT vis-à-vis DWT, it is imperative to employ Dual Tree - Complex Wavelet Transform (Selesnick) (DT-CWT(S)) for this work.

The DT-CWT(S) algorithm is used to design and implement the Dual Tree structure (up to Level 2) using MATLAB, employing first and second stage low and high pass filter coefficients given by Selesnick. The impulse responses of three 2-D sub-bands (2-D non separable filters for detailed coefficients) of DWT and six sub-bands (2-D non separable filters for detailed coefficients) of DT-CWT are shown in Figure 9. 


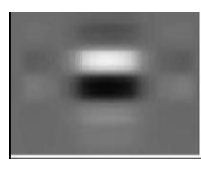

$+$
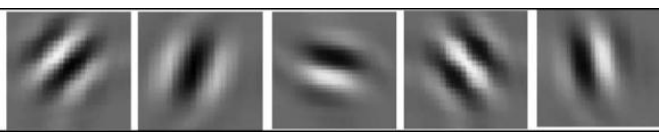

Figure 9. Impulse responses of sub-bands of DWT and DT-CWT.

DT-CWT has 06 directional wavelets oriented at angles of $\pm 15, \pm 45, \pm 75$ in 2 -Dimension. We get these six directions by passing the 2-D signal (image) through a real tree structure using the filter coefficients of both real and imaginary trees. The wavelet coefficients of each image which formed part of the Training Database were thus obtained in all the six directions and stored for further matching and testing. These directions can be seen clearly from the Figure 10 which represents the Level 1 decomposition of an image.
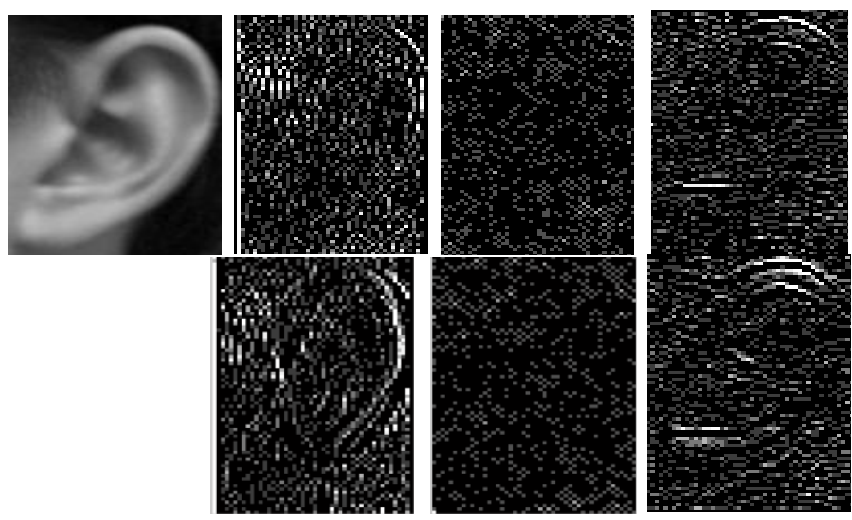

Figure 10. Real and imaginary tree wavelet sub-band images

\section{EXPERIMENTAL RESULTS}

All the Training images of both the databases (MCTE database of 240 images of 40 subjects for right and left ears and UND database of 219 subjects under J-collection and Gcollection) are processed and their respective wavelet coefficients at Level 1 and Level 2 are calculated. Energy, Entropy, Mean and Standard Deviation of each image's wavelet coefficient are then calculated and stored in an MS Access database. Thereafter images from the Test Set and random images were matched with these stored values using Euclidean and Canberra distance matching techniques and results for False Acceptance Rate (FAR), False Rejection Rate (FRR), Equal Error Rate (EER) and Receiver's Operating
Curve (ROC) compiled at various thresholds. All the results are stated in Table 1.

Figure 11 to 14 shows the FAR, FRR and ROC of best and worst case of Canberra distance and best and worst case of Euclidian distance when tested on database with following details.

Name Of Data-Base : UND - Collection G

No of Images in the Training database: 20

No of Images in Test Database: 90
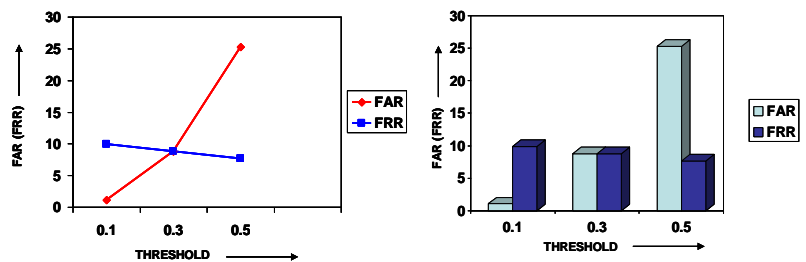

RECEIVER'S OPERATING CURVE

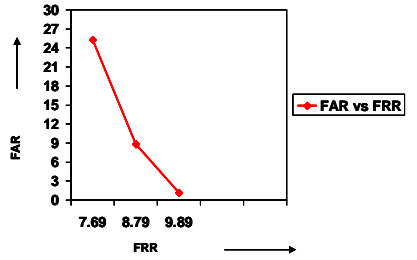

Figure 11. Results using Canberra distance and feature vector of energy only (Worst case of Canberra Distance)

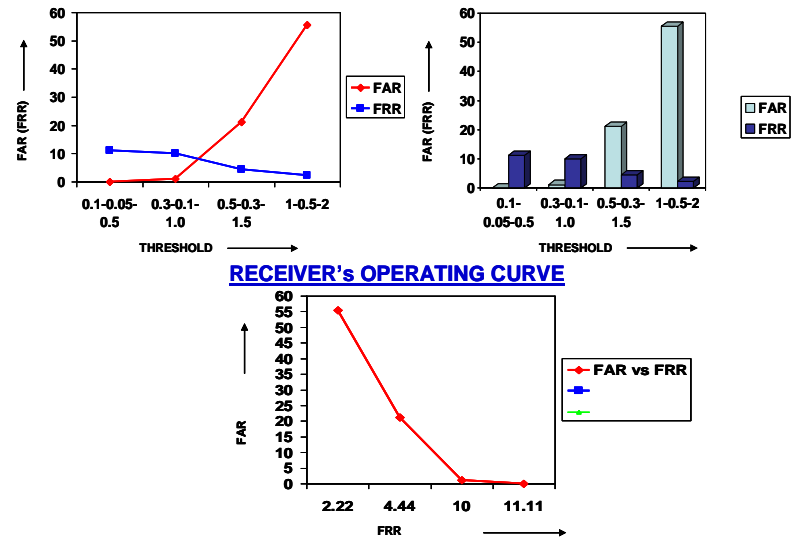

Figure 12. Results using Canberra distance and feature vector of energy + std deviation + entropy (Best case of Canberra Distance)

Table 1. Compiled results of Avg FAR, Avg FRR and Avg Recognition rate using Canberra and Euclidian distance for different feature vectors

\begin{tabular}{|l|c|c|c|c|c|c|}
\hline \multirow{2}{*}{ Feature Vector / Distance } & \multicolumn{2}{|c|}{ Canberra Distance } & \multicolumn{3}{c|}{ Ecludian Distance } \\
\cline { 2 - 6 } & Avg FAR & Avg FRR & Recognition Rate & Avg FAR & Avg FRR & Recognition Rate \\
\hline Energy only & $8.79 \%$ & $8.79 \%$ & $91.21 \%$ & $16.66 \%$ & $5.55 \%$ & $83.34 \%$ \\
\hline
\end{tabular}




\begin{tabular}{|l|c|c|c|c|c|c|}
\hline Entropy only & $6.59 \%$ & $8.79 \%$ & $93.41 \%$ & $12.22 \%$ & $6.66 \%$ & $87.88 \%$ \\
\hline Standard Deviation only & $8.79 \%$ & $8.79 \%$ & $91.21 \%$ & $21.11 \%$ & $4.44 \%$ & $78.89 \%$ \\
\hline Energy+ Entropy & $3.29 \%$ & $8.79 \%$ & $96.71 \%$ & $3.33 \%$ & $8.88 \%$ & $96.67 \%$ \\
\hline Energy+ Standard Deviation & $5.49 \%$ & $4.39 \%$ & $94.51 \%$ & $4.44 \%$ & $6.66 \%$ & $95.56 \%$ \\
\hline $\begin{array}{l}\text { Entropy+ Standard Deviation } \\
\text { Energy + Entropy + Standard } \\
\text { Deviation }\end{array}$ & $\mathbf{2 . 1 9 \%}$ & $\mathbf{7 . 6 9 \%}$ & $\mathbf{9 7 . 8 1 \%} \%$ & $3.33 \%$ & $7.77 \%$ & $96.44 \%$ \\
\hline
\end{tabular}
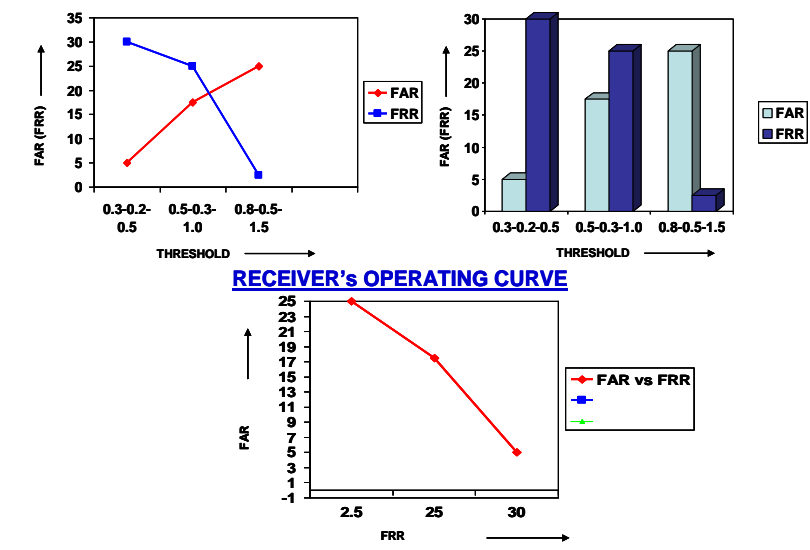

Figure 13. Results using Euclidian distance and feature vector of Std Deviation only (Worst case of Euclidian Distance)
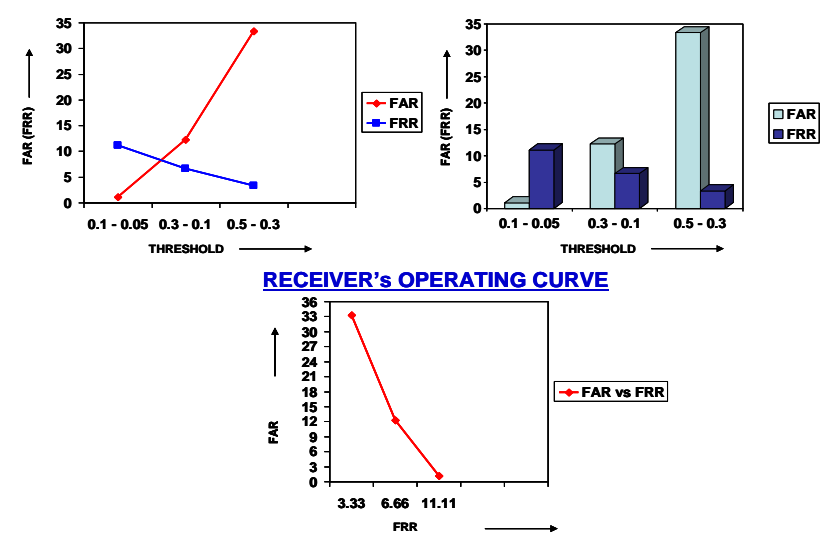

Figure 14. Results using Euclidian distance and feature vector of energy + std deviation + entropy (Best case of Euclidian Distance)

The maximum recognition rate of $81 \%$ is obtained when DWT is used for feature extraction and Canberra distance is used as similarity metric for combined vector of energy, std. deviation and entropy. FAR, FRR and ROC for it is shown in figure 15 .

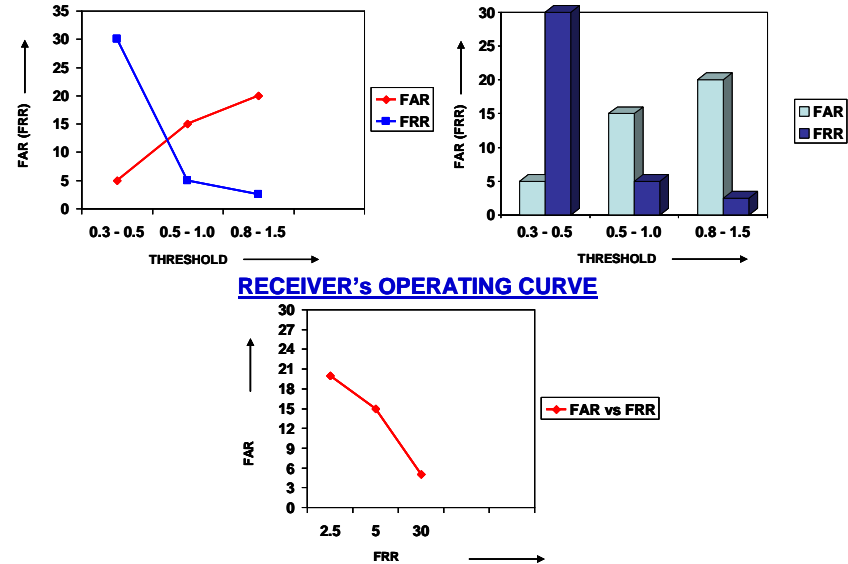

Figure 15. Results of DWT using Canberra distance and feature vector of energy + std deviation + entropy (Best case of DWT)

\section{CONCLUSIONS}

The authors have introduced a new 2D DT CWT for ear recognition first time because of it's ability to capture shift invariant features in 06 orientations. The experimental results have demonstrated the effectiveness of the proposed method in terms of improving the recognition rate.

Canberra distance has shown better results than Euclidian distance because it normalises the individual feature components before finding the distance between the two images.

The best recognition rate of over $97 \%$ has been achieved using Canbera distance when feature vectors of energies, standard deviation and entropy of sub-bands of DT-CWT are used together.

The authors are working on improving the recognition rate by using the RCWF [22], [23], [24] in combination with DTCWT to obtain features in 12 orientations (06 by DT-CWT and 06 by RCWF).

\section{REFERENCES}

[1] Alfred Iannarelli, "Ear Identification," Forensic Identification Series, Paramont Publishing Company, Fremont, California, 1989.

[2] K. Chang., K.W. Bowyer, S. Sarkar, and B. Victor, "Comparison and Combination of Ear and Face Images in Appearance-Based Biometrics," IEEE Transactions on Pattern Analysis and Machine Intelligence, vol. 25, no. 9, pp. 1160-1165, September 2003. 
[3] D.J. Hurley, M.S. Nixon, j.n. Carter, "A New Force Field Transform for Ear and Face Recognition," Proc. IEEE 2000 International Conference on Image Processin (ICIP 2000), IEEE Press, , pp. 25-28.

[4] B. Victor, K.W. Bowyer,S. Sarkar, "An evaluation of face and ear biometrics" Proc. International Conference on Pattern Recognition, August 2002, pp. 429-432.

[5] B. Moreno, A. Sánchez, J.F. Vélez., "On the Use of Outer Ear Imagę̧ for Personal Identification in Security Applications," Proc. IEEE 33 Annual International Carnahan Conference on Security Technology, 1999, pp. 469-476.

[6] M. Burge and W. Burger, "Ear biometrics in computer vision," Proc. International Conference on Pattern Recognition, (ICPR' 00), vol. 02, 2000, pp. 822-826.

[7] D. J. Hurley, M. S. Nixon, and J. N. Carter, "Automatic ear recognition by force field transformations," Proc. IEE Colloquium: Visual Biometrics, 2000, pp. 8/1-8/5.

[8] H. Chen and B. Bhanu, "Human ear detection from side face range images," Proc. International Conference on Pattern Recognition, (ICPR' 04), vol. 3, IEEE Computer Society Press, 2004, pp. 574-577.

[9] L. Alvarez, E. Gonzalez, and L. Mazorra, "Fitting ear contour using an ovoid model," in IEEE International Carnahan Conference on Security Technology, (ICCST’ 05), 2005, pp. 145-148.

[10] S. Ansari and P. Gupta, "Localization of ear using outer helix curve of the ear," Proc. ( International Conference on Computing: Theory and Applications, ICCTA' 07), , IEEE Computer Society Press, 2007, pp. 688-692.

[11] L. Yuan and Z.-C. Mu, "Ear detection based on skin-color and contour information," Proc. International Conference on Machine Learning and Cybernetics, (ICMLC' 07), vol. 4, (Hong-Kong), 2007, pp. 2213-2217,

[12] Hui Chen and Bir Bhanu, "Human Ear Recognition in 3D", IEEE Transaction on Pattern Analysis and Machine Intelligence, Vol 29, No 4, April 2007

[13] Surya Prakash, Umarani Jayaraman and Phalguni Gupta, "Ear Localization from Side Face Images using Distance Transform and Template Matching", Proc. IEEE Int'l Workshop on Image Processing Theory, Tools and Applications, (IPTA 2008), Sousse, Tunisia, Nov 2008 pp. 1-8.

[14] D. Hurley, M. Nixon, and J. Carter, "Force Field Energy Functionals for Ear Biometrics" Computer Vision and Image Understanding, vol.98, 2005, pp.491-512.
[15] Ping Yan and Kevin W. Bowyer, "Biometric Recognition Using 3D Ear Shape" IEEE Transactions on pattern analysis and machine intelligence, vol. 29, no. 8, August 2007.

[16] A. Sana, F. Gupta and R. Prukait, "Ear Biometrics: A new approach.," Proc. Sixth International Conference on Advances in Patern Recognition, Jan 2007, pp.46-50.

[17] Xiaoyun Wang and Weiqi Yuan, "Gabor wavelets and General Discriminant analysis for ear recognition ," Proc. 8th World Congress on Intelligent Control and Automation (WCICA 2010), IEEE Press, 2010 , pp. $6305-6308$

[18] L. Gutierrez, P. Melin, and M. Lopez, "Modular neural network integrator for human recognition from ear images ," Proc. International Joint Conference on Neural Networks (IJCNN 2010), IEEE Press, 2010, pp. 1-5.

[19] Wang Xiaoyun and Yuan Weiqi, "Human ear recognition based on block segmentation ," Proc. International Conf on Machine Learning and Cybernetics, 2009 , vol. 4, pp. $2418-2422$.

[20] Ivan W. Selesnick, Richard G. Baraniuk, and Nick G. Kingsbury, "The Dual Tree Complex Wavelet Transform: A Coherent Framework for Multiscale Signal and Image Processing", IEEE Signal Processing Magazine, Nov 2005, pp. 123-151.

[21] N.G. Kingsbury, "The dual-tree complex wavelet transform: A new technique for shift invariance and directional filters," in Proc. 8th IEEE DSP Workshop, Utah, Aug. 9-12, 1998, paper no. 86.20.

[22] M. Kokare, P.K. Biswas, and B.N. Chatterji, "Rotation invariant texture features using rotated complex wavelet for content based image retrieval,” in Proc. IEEE Int. Conf. Image Processing, Singapore, Oct. 2004, vol. 1, pp. 393-396.

[23] Rajesh Bodade and Sanjay Talbar, "Iris Recognition Using MultiDirectional Wavelets: A Novel Approach" Journal of Advances in Engineering Sciences Sect. C (3), July-September 2008, (special issue on Image Processing) ISSN: 0973-9041

[24] R. Bodade and S. Talbar, "Iris Recognition using Combination of Dual Tree Rotated Complex Wavelet and Dual Tree Complex Wavelet" Proc. IEEE International Conference on Communication-2009 (ICC-2009), IEEE Press , June 2009.

[25] J. Daugman, "High Confidence Visual Recognition of Persons by a Test of Statistical Independence," IEEE Trans. PAMI, vol. 15 , no. 11, Nov. 1993, pp. 1148-1161. 